\title{
DESIGN CONSIDERATION OF A MULTI-FUNCTION OTOACOUSTIC EMISSION MEASUREMENT SYSTEM
}

\author{
Minghui Du ${ }^{1}$, F. H.Y. Chan ${ }^{2}$, F.K. Lam ${ }^{2}$, and Jun Ren ${ }^{1}$ \\ ${ }^{1}$ Department of Electronic and Telecommunication Engineering, \\ South China University of Technology, Guangzhou, China \\ ${ }^{2}$ Department of Electrical and electronic Engineering, \\ The University of Hong Kong. Hong Kong, China. \\ mhdu@eee.hku.hk
}

\begin{abstract}
A new approach for recording otoacoustic emissions(OAEs) is described in the paper. The system is based on a personal computer equipped with INTEL Pentium CPU. A single chip microcomputer INTEL 8096 is developed to be a stimulus generator. Some critical problems and circuit parameters in the design of this system are presented in the paper. The main advantages of the system are (1) it can record three kinds of OAEs which have many clinical applications; (2) it can save raw data for further analysis, as most researchers need; (3) plenty of analysis functions can be developed in this system. With the use of the newly developed system, SOAEs, TEOAEs, and DPOAEs have been successfully measured.
\end{abstract}

\section{INTRODUCTION}

Since the exciting discovery of otoacoustic emission[1], it has now been accepted that the otoacoustic emissions in the ear canal represent an active non-linear micro mechanism within the cochlea. As described in [2] that different types of otoacoustic emissions exist.
According to types of stimulus, OAEs can be classified as spontaneous OAE (SOAE), transient evoked OAE (TEOAE), stimulus-frequency OAE (SFOAE), and distortion product OAE (DPOAE). Due to the limited applications of SFOAE, little attention has been paid to this kind of OAE.

Up to date, several kinds of OAE measurement apparatus are available in the market[3-4]. Most of them measure either TEOAEs or DPOAEs. For the purpose of physiological research and clinical usage, we developed a multi-function OAE measurement system which not only can measure TEOAE, but also DPOAE and other kinds of OAEs. It not only can give the measurement results, but also keep or store the raw data for further processing. Its function can be easily expanded. New algorithms can also be implemented in the system for research.

\section{SYSTEM DESIGN}

\section{A. Block Diagram}

The instrumentation system, shown in fig.1, is based on a personal computer equipped with INTEL

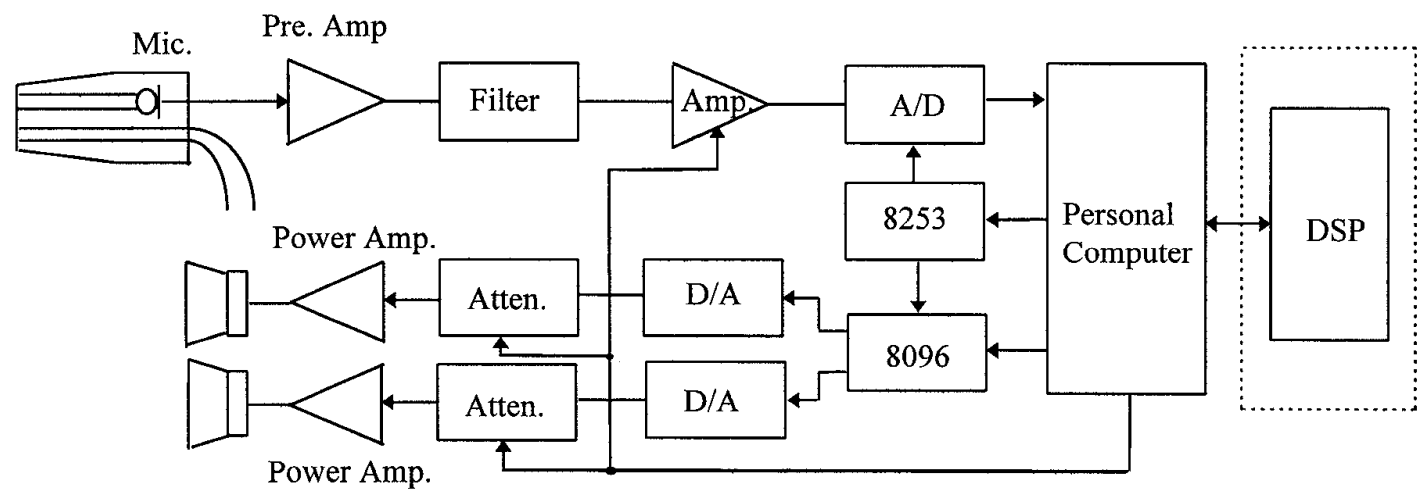

Fig.1 Block diagram of the OAE measurement system 
PENTIUM CPU. Another single chip microprocessor INTEL 8096 is used for generating stimulus. Data are collected and processed by the personal computer, and the results and raw data can be stored on disk. [5]

\section{B. Transducer}

The principal component of the OAE measurement system is an acoustic probe which incorporates a sensitive miniature microphone for recording acoustic signal, and a means for presenting stimuli. Because the vast majority of emission represents extremely lowlevel responses, the sensitivity and noise floor of the microphone are of critical importance in determining the quality of OAE-measuring system. Microphone sensitivity is determined by both its design, with respect to the surface and mass of the microphone membrane, and, in the case of condenser microphone, the amount of voltage applied to the microphone.

For DPOAE measurement, it is important to minimize cross-talk between the stimulus channels, which can lead to generation of intermodulation distortion. For this reason, it is advantageous to place the loud speakers outside the probe, and to use speakers with a high source impedance. The two speakers must be acoustically isolated from each other. However, for TEOAEs, it is more appropriate to have an integrated miniature assembly of probe together with speaker, because this setup enhances transient response and frequency bandwidth.

In our first experiment, we chose a probe provided by Tianjing Audiophone Factory, Tianjing, China, and it has a flat frequency response between 0.2 to $10 \mathrm{KHz}$, and linear operation up to $100 \mathrm{~dB}$ SPL.

\section{Stimulus Generation}

A microprocessor is used to generate stimulus. Stimuli are generated digitally with software. They are sent out via 16 -bit $\mathrm{D} / \mathrm{A}$ converters at $50 \mathrm{KHz}$ rate. These analogue signals are then fed to a digitally controlled attenuator. The attenuated signals are passed to speakers via power amplifiers.

The stimulator provides intensity levels in the range -10 to $90 \mathrm{~dB}$ SPL. The stimulus level is adjusted automatically by the computer after positioning the probe in the ear canal, based on probe feedback from microphone output signal level.

The stimulus signal can be click, tone burst, pure tone, maximum length sequence, and so on. One can generate custom stimuli by programming the 8096 single chip computer.

\section{Amplifiers and Filters}

The pre-amplifier is an instrumentation amplifier with a fix gain of 10000 , followed by a band pass filter from 500 to $10000 \mathrm{~Hz}$. A programmable amplifier stage, capable of adding an extra gain of 100 , is inserted. The amplified and filtered signal is passed to a variable gain amplifier controlled by the main computer. The gain can be adjusted from 1 to 100 . This enables auto-ranging and ensures that the A/D converter is always used efficiently.

\section{E. Data Acquisition.}

In order to measure TEOAE and DPOAE, timelocked averaging technique is needed. Therefore, it is important to ensure the synchronization of stimulating and data acquisition. For this purpose a timer/counter INTEL 8253 is used, so that the two clock signals for A/D and D/A converters are at a fixed phase relationship. This insured that the resulting waveforms from every recorded sweep are synchronized.

The dynamic range of microphone, amplifier circuits and the A/D converter is also important. In order to measure DPOAEs in impaired ears, which is usually as much as $90-100 \mathrm{~dB}$ below the stimulus tone level, a 16-bit $\mathrm{A} / \mathrm{D}$ converter was chosen so that the dynamic range approaches $96 \mathrm{~dB}$.

Data were acquired at the sampling rate of $40 \mathrm{kHz}$ for stimulus frequency higher than $2000 \mathrm{~Hz}$. Otherwise, a sampling rate of $20 \mathrm{kHz}$ was used.

\section{F. Linearity}

The linearity of the microphone and the preamplifier circuit is important. Although we can find some microphones with higher sensitivity, many of them are nonlinear at a higher evoking-stimulus level. For DPOAE measurement, no component of the stimulus-generation or response-collection circuits should contain a non-linearity that will generate $2 f_{1}-f_{2}$ distortion products, which may be mistaken for DPOAEs originating from the cochlea. For TEOAE, the measurement circuit is required to be linear since it is standard practice to utilize the characteristic compressively nonlinear growth of the TEOAEs with stimulus level to increase the reliability of TEOAE measurement.

The non-linearity of the amplifier and filter system is well below $0.01 \%$ at frequency range from $500-10000 \mathrm{~Hz}$.

\section{G. Software System}

The software system for OAE measurement contains mainly 4 modules, as shown in fig. 2 . It is developed on Microsoft WINDOWS platform.

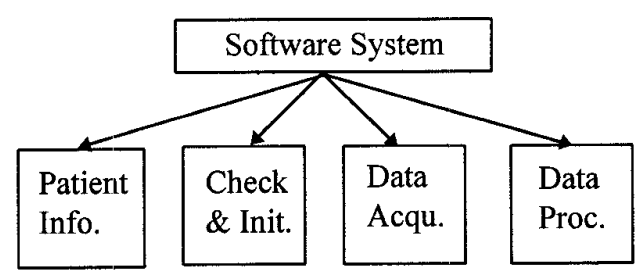

Fig.2 Modules of the software system 
The "check \& initialization" module mainly implements checking ear-probe fit, stimulus stability, and initializes parameter for stimulating and collecting. "Data acquisition" module implements the hardware control for data collection, such as presenting stimulus, sampling, storing raw data. The "data processing" module contain programs for signal processing and analysis, such as timelocked averaging, FFT spectrum estimation, adaptive filtering, correlation analysis, OAE interpretation, etc.

\section{H. Some Specific Algorithms}

Many classical algorithms were implemented in this system for OAE processing. As argued by [6] that synchronous averaging could improve the signal to noise ratio by a factor of $\mathrm{K}^{1 / 2}$, where $\mathrm{K}$ is the number of segments averaged, we incorporated the measurement of synchronous spontaneous OAE and synchronous DPOAE to the system. Synchronous SOAEs (SSOAEs) are measured by presenting 80 micro-second duration clicks at a rate of about $10 \mathrm{~Hz}$. Although the clicks will also evoke TEOAEs, these decay within about $20 \mathrm{~ms}$, and the response is dominated by SSOAEs which are entrained by the clicks and persist over the entire sampling period.

Maximum length sequence technique [7-8] is also provided in this system. This technique can make a significant improvement to the recording of OAEs[9]. It is shown in our previous paper[7-8] that it will improve the measurement signal to noise ratio by a factor of $(\mathrm{L}+1)^{2} / 4 \mathrm{~L}$, where $\mathrm{L}$ is the length of maximum length sequence, so that the measurement time could be shortened. In practice, this technique can give a speed improvement in excess of the factors of 2 to 5 .

Not only the spectral domain DPOAEs can be measured with this system, but also time domain parameters. DPOAEs exhibit a distinct latency, which is a common phenomenon of all forms of transient-evoked OAEs. The DPOAE delay is an important quantity because it conveys unique information about the cochlea traveling wave progression. To measure the latency of DPOAEs, phase gradient method [10] can be employed .

\section{RESULTS AND DISCUSSIONS}

\section{A. SOAE Measurement}

A probe was placed in the ear canal of the subject who was seated in a soundproof room. Signal from the probe was amplified, filtered, and then served as input to the $\mathrm{A} / \mathrm{D}$ converter. Data were sampled at a $20 \mathrm{kHz}$ rate. 4096 point FFT (the frequency resolution is about $5 \mathrm{~Hz}$ ) was used to analyze the Hanning windowed signals, and averaging technique was then applied to all estimated spectrum. The measured SOAEs is shown in fig. 3 .

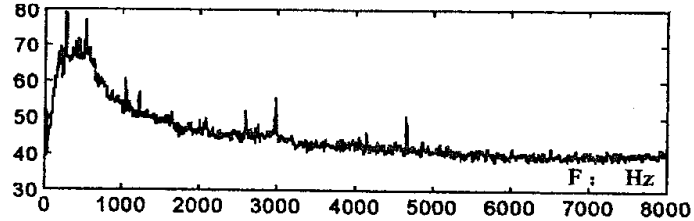

Fig 3. An averaged spectrum which contains SOAEs.

\section{B. TEOAE Measurement}

A TEOAE of a normal ear is measured, shown in fig.4. Fitting the probe is important for making the measurement. Data were collected in a time window of 2.5 to $20 \mathrm{~ms}$ post-stimulus. Time-locked averaging was applied to all these windowed signals (usually over 100 responses, dependent on the stimulus intensity). TEOAE in fig. 4 is a result of 300 response averaging. The two independently obtained waveforms demonstrate the repeatability of the TEOAE of the subject.

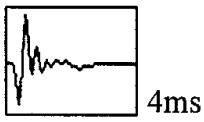

(a)

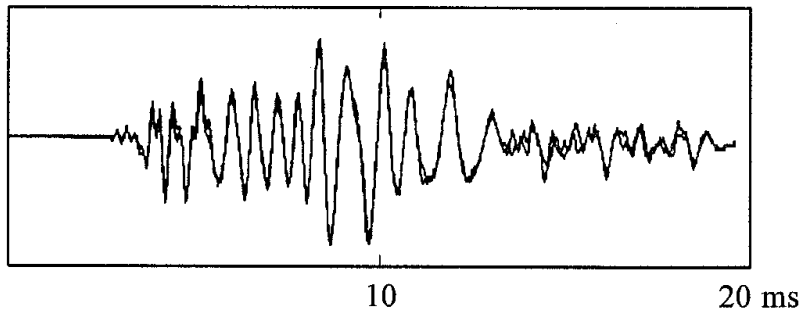

(b)

Fig.4 A TEOAE measurement result.
(a). stimulus
(b). TEOAE

\section{DPOAE Measurement}

The subject was seated in a comfortable chair placed inside a soundproof room, and was instructed to remain quiet and alert during the recording. DPOAEs were recorded at a stimulus intensity about $60 \mathrm{~dB}$ SPL. The two stimuli were presented at equal intensities with a frequency ratio of approximately 1.2 to 1.3 .

Data were accumulated at a sampling rate of 20 $\mathrm{kHz}$. The stimuli were presented for $100 \mathrm{~ms}$, and the raw signal were sampled 2048 points for each trial. 200 sweeps were applied, and the averaged data were finally processed by a 2048 points FFT algorithm, using Hanning window. A DPOAE and primary stimulus energy in spectral domain is shown in fig. 5. 


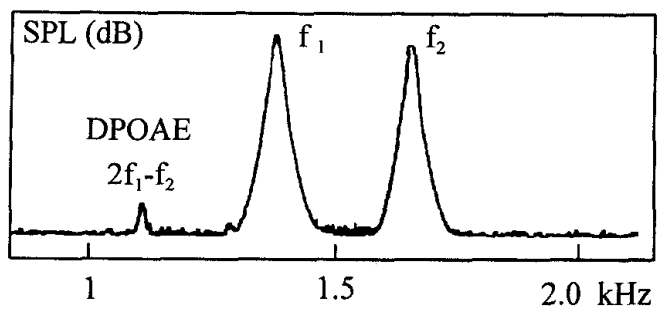

Fig. 5 DPOAE measurement result

It is seen that the system can give fairly good OAE measurement. Due to the different sensitivity of the microphone and the number of trial of averaging, difference in estimates of OAEs from our study to other studies are expected to exist. Therefore, calibrations and comparative study might be needed in future works.

During measurement experiments, we have found that it is important to fit the probe within the ear canals of subjects. A good ear-probe fit effectively attenuates most background noise, moreover, if the fit is loose, the waveform of the recorded OAEs will be inconsistent.

\section{CONCLUSION}

OAEs have been a standard technique in audiology clinics around the world. Though current OAE measurement apparatus provides useful information, it is clear that these instruments can be improved.

The most difficult development of this system has been the very low signal to noise ratio in $\mathrm{OAE}$ measurement. To overcome this difficulty, probe fitting, as well as linearity, dynamic range, noise level of the electronic circuits are some of the important points in the design. Time domain averaging technique can also improve signal to noise ratio. Therefore, a synchronizing circuit is also incorporated to the system.

The present system with multi-functions has been shown to be an efficient instrument for recording OAEs. The system can record SOAE, TEOAE and DPOAE. It can also save raw data for further processing. We believe that, after further refinement and development, this system will prove to be a powerful tool for diagnosis and research in the near future.

\section{ACKNOWLEDGMENT}

This work was supported by Guangdong Natural Science Foundation (No. 960316), Guangdong Province, China. Thanks also due to Prof. P.W.F. Poon from the Department of Physiology, National Cheng Kung University, Taiwan, China, for his constructive suggestions on this work. We also thank voluntary subjects for their helps in experiments.

\section{REFERENCE}

[1] D.T. Kemp, Stimulated Acoustic Emissions from Within the Human Auditory System, J. Acoust. Soc. Am., 64:1386-1391, 1978

[2] R. Probst, B.L. Lomnsbury-Martin, G.K. Martin, A Review of Otoacoustic Emissions, J. Acoust. Soc. Am., 89(5):2027-2065, May, 1991.

[3] M.L Whitehead, B.B. Stagner , B.L. LomnsburyMartin, G.K. Martin, Measurement of Otoacoustic Emission for Hearing Assessment. IEEE Engineering in Medicine and Biology, April/May 1994. 210-226.

[4] A. Gluzmann, H. Kunov, On the use of a 16-bit Multimedia Sound board in a virtual Instrument for Hearing Assessment, 1995 IEEE EMBS 17th annual conference, Montreal, Canada, Sept. 1995.

[5] Sun Junliang, The development of OAE measurement system. Master Degree Thesis, South China University of Technology. March 1998.

[6] R. S. Kulik, H. Kunov, Results of two types of averaging for distortion product otoacoustic emission measurement. 1995 IEEE EMBS 17th annual conference, Montreal, Canada, Sept. 1995

[7] F.H.Y. Chan, F.K. Lam, P.W.F. Poon, M.H. Du., Measurement of human BAERs by the maximum length sequence technique, Medical \& Biological Engineering \& computing, 30(1): 32-40, Jan. 1992

[8] Du Minghui, Xu Bingzheng, F.H.Y. Chan, F.K. Lam, P.W.F. Poon, Auditory Brainstem Evoked Potential measurement by M-sequence. Chinese Journal of Biomedical Engineering. 15 (3):214-221, Sept. 1996:

[9] A.R.D. Thornton, Click-evoked otoacoustic emissions: new techniques and applications, British Journal of Audiology, 27, 109-115, 1993.

[10]C. F. O Mahoney, D.T. Kemp, Distortion product otoacoustic emission delay measurement in human eras, J. Acoust. Soc. Am. 97(6), June 1995, 37213735 . 